\title{
EDUCACIÓN SUPERIOR EN CHILE Y SU INTERNACIONALIZACIÓN ${ }^{1}$
}

\author{
Dr. Jaime Caiceo Escudero
}

\section{RESUMEN:}

A lo largo de toda la historia (desde la universidad medieval hasta nuestros días) la educación superior ha pretendido la universalización de su quehacer. Justamente porque universidad es universalización. En la época de la globalización, ello se transforma en algo imprescindible. Chile no ha estado ajeno a ello y aquí se presentan algunos antecedentes al respecto.

Palabras claves: Educación Superior/ Universidad/ Internacionalización de la educación.

\section{HIGHER EDUCATION IN CHILE AND THEIR INTERNATIONALIZATION}

\begin{abstract}
:
Throughout History (from the medieval university to the present), Higher Education has sought the universalization of its work; due to the fact that university is considered as universalization. In the era of globalization, this fact becomes something essential. Chile has been part of this fact and the background to this matter is presented.
\end{abstract}

Keywords: Higher education/ University/ Internationalization of education.

\section{I.- Introducción}

Agradezco la invitación a las autoridades de la Universidad Nacional de Cuyo por la invitación a participar en este interesante Foro-Panel con una temática tan propia de los nuevos tiempos.

En efecto, la internacionalización de la educación superior "[...] es el proceso de entrega de una dimensión internacional, intercultural o global a los propósitos, funciones y programas de Educación Superior" (www.mineduc.cl). Ello trae diversas consecuencias positivas para las personas de los países participantes en una primera etapa y, para los países en su conjunto, en una segunda etapa. Entre las principales ventajas, se pueden indicar:

1.- "[...] significativos beneficios económicos, de comercio y diplomáticos porque los estudiantes becados en el extranjero mantienen relaciones privilegiadas con los países en que estudiaron durante todas sus carreras".

2.- "El intercambio académico permite una circulación más rápida y una diseminación de los resultados de investigación producida en otra parte, y le da ímpetus a la investigación y a la innovación que de otra manera ocurre en forma aislada".

3.- "La Internacionalización de la Educación Superior contribuye a la eficiencia del sistema de investigación y por extensión a la capacidad de innovación nacional".

4.- "Aumentan las oportunidades para compartir costos al juntar expertos que vienen de distintos centros de investigación".

5.- "La Internacionalización contribuye al multiculturalismo y despierta el interés por culturas más allá de las fronteras (efecto de la presencia de estudiantes extranjeros y aumento de contenido internacional en programas de educación superior)" (www.mineduc.cl). 
Esta participación se divide en tres partes: Descripción de la Educación Superior en Chile; principales convenios existentes entre universidades nacionales y extranjeras o entre países y un esbozo de las principales estrategias usadas para internacionalizar las universidades locales.

Para realizar esta presentación se recurrió a la legislación vigente, a entrevistas realizadas a personal de la División de Educación Superior del Ministerio de Educación y al material existente en algunas universidades en las páginas web de las mismas, en la de la Asociación de Universidades del Consejo de Rectores y del propio Ministerio de Educación.

\section{II.- Descripción de la Educación Superior en Chile}

Durante la colonia se fundaron dos universidades católicas, dependientes de la Orden de Santo Domingo - Universidad Santo Tomás - y de la Compañía de Jesús Universidad de San Miguel-, respectivamente, comenzando a operar en 1622 y una universidad dependiente de la corona -la Real Universidad de San Felipe- en 1747.

Con el comienzo de la República se fundaron en Santiago la Universidad de Chile en 1842 -universidad pública y de inspiración laica- y la Universidad Católica de Chile en 1888 -universidad particular dependiente de la Iglesia Católica y como reacción al laicismo imperante en la universidad pública-; durante el siglo XX y hasta antes de la dictadura militar se fundaron 6 universidades más, tanto en Santiago como en Provincias: La Universidad de Concepción -universidad particular dependiente de la masonería, instalada en la ciudad de Concepción- en 1920; la Universidad Técnica Federico Santa María universidad particular laica, instalada en la ciudad de Valparaíso- en 1926; la Universidad Católica de Valparaíso -universidad particular dependiente de la Iglesia Católica, instalada en la ciudad de Valparaíso - en 1928; la Universidad Técnica del Estado -universidad pública originada a partir de la Escuela de Artes y Oficios fundada en 1849 en Santiago- en 1947, la Universidad Austral de Chile -universidad particular laica, instalada en la ciudad de Valdivia- en 1954 y la Universidad Católica del Norte -universidad particular dependiente de la Iglesia Católica, radicada en la ciudad de Antofagasta- en 1964. En resumen, hasta antes del golpe militar, existían en el Chile Republicano dos universidades públicas, las cuales habían ido creando sedes regionales en diferentes ciudades a lo largo del país, tres universidades católicas particulares -la de Santiago también tenía algunas sedes en el sur de Chile- y tres universidades laicas particulares. Todas estas universidades recibían aporte fiscal cercano al $90 \%$ de su presupuesto para financiar sus actividades; ello comenzó a disminuir con la dictadura militar, llegando a recibir aportes públicos no superiores al 30\% de su presupuesto, política que se mantuvo e incluso se restringió más durante los 20 años de gobiernos democráticos.

En 1981, la dictadura militar reformuló la educación superior en Chile, ubicando en tal rango a las universidades, los institutos profesionales y los centros de formación técnica. En esta ocasión sólo se abordará el tema universitario. A partir del DFL 1 del año indicado precedentemente, se autoriza la creación de universidades privadas, bajo el amparo de la libertad académica (art. $5^{\circ}$ del DFL $\mathrm{N}^{\circ} 1$ ), independientemente de las 8 existentes; las universidades serán las únicas instituciones que darán grados académicos (Licenciatura, Magíster y Doctorado, Art. $9^{\circ}$ del DFL $N^{\circ} 1$ ) y títulos profesionales de las 12 carreras que en esa oportunidad se indicaron (Art. 12 $2^{\circ}$ del DFL $\left.N^{\circ} 1\right)^{3}$. De las dos universidades públicas surgieron las universidades públicas regionales, manteniéndose las originales sólo en Santiago, la Universidad de Chile y la Universidad de Santiago de Chile (a la Técnica del Estado se le cambió el nombre); a ellas se unieron las 6 restantes más 
algunas nuevas universidades católicas regionales desprendidas de la Universidad Católica de Chile ${ }^{4}$. Las 8 universidades antiguas más las nuevas surgidas de ellas mismas se reformuló el Consejo de Rectores con un total de 25 entidades $^{5}$, recibiendo todas ellas el nombre de universidades tradicionales (Fuente: División de Educación Superior, Mineduc).

Las nuevas universidades privadas no recibirían aportes fiscales directos para su funcionamiento; sólo el aporte fiscal indirecto (AFI) creado para estimular con un aporte a aquellas instituciones de educación superior que tuvieran matriculados en sus aulas a alumnos de los 27.000 mejores puntajes de la Prueba de Aptitud Académica -PAA-, hoy Prueba de Selección Universitaria -PSU-; esta prueba la rinden todos los años los estudiantes que deseen ingresar a las universidades del Consejo de Rectores. Desde 1981 en adelante se han fundado más de 40 universidades privadas, algunas de las cuales ya han desaparecido; están vigentes en la actualidad $36^{6}$; la mayoría de ellas la han fundado inversionistas, salvo algunas institucionales, dependientes de Congregaciones Religiosas Católicas o Evangélicas. Para fundar una universidad se requiere la aprobación del Ministerio de Educación, previa constitución como persona jurídica de derecho privado sin fines de lucro (Art. $15^{\circ}$ del DFL No 1 ).

\section{III.- Principales convenios existentes entre universidades nacionales y extranjeras o entre países}

La Declaración de Bolonia de 1999 incentiva a los países latinoamericanos, en este caso a Chile, para que estudiantes chilenos puedan estudiar en Europa y se beneficien de lo que significa tener un título o grado en Europa: Tendrán los mismos beneficios de un europeo, es decir, un título reconocido y compatible con la estructura de los estudios superiores de 46 países europeos, pudiendo pasar de un ciclo a otro sin necesidad de trámites de homologación. De allí la importancia de que universidades chilenas firmen convenios de intercambio con universidades europeas.

Tal como se indicó anteriormente, en Chile existen universidades tradicionales y universidades privadas nuevas. Las primeras conforman el Consejo de Rectores y ellas, en su conjunto han logrado convenios de intercambio internacional, tanto para sus alumnos como para sus académicos con diferentes países; la mayoría de estos Convenios tienen una duración de 5 años renovables. Entre ellos, cabe mencionar los existentes con (www.cruch.cl):

1.- Alemania: Con este país existen dos importantes convenios de cooperación académica:

a) El Convenio Marco Chileno Alemán con la Asociación de las Universidades (CRUCH) y otras Instituciones de Educación Superior de Alemania HOCHSCHULREKTORENKONFERENZ (HRK) fue suscrito el 16 de abril de 1999. Este acuerdo fue complementado en el año 2000 con un convenio adicional con el objeto de considerar el desarrollo de los sistemas de Educación Superior de ambos países. Posteriormente, el 26 de noviembre de 2002 se firmó una declaración conjunta entre ambas instituciones sobre la supervisión Binacional de Tesis Doctorales. En el marco de este Convenio de Cooperación con la Asociación de las Universidades y otras instituciones de Educación Superior de Alemania el 5 de octubre de 2006 se realizó en Munich - Alemania un Seminario Bilateral HRK-CRUCH, que dio como resultado la firma de una Declaración Conjunta de intenciones para fortalecer las relaciones bilaterales de apoyo a los programas de movilidad académica y estimular la creación de un Colegio Doctoral que 
pueda ofrecer a los alumnos la obtención de un grado académico conjunto de Doctorado $(\mathrm{PhD}) \mathrm{y} / \mathrm{o}$ doble titulación entre ambos sistemas universitarios. Las conversaciones en torno a la creación del Colegio Doctoral Chile-Alemania comenzaron el 20 de mayo de 2008, y se consideró la creación de hasta seis Colegios Doctorales, según las áreas temáticas de prioridad acordadas entre el HRK y el CRUCH para establecer y desarrollar la colaboración académica conjunta y favorecer la formación plena de los doctorandos. El 28 de agosto de 2008 el Consejo de Rectores en pleno aprobó el texto del convenio y fue enviado a sus pares en Alemania para su revisión y aprobación; finalmente, el pasado 9 de Marzo de 2009, se celebró en la ciudad de Santiago la firma del convenio entre el HRK y el CRUCH.

b) Un Programa de Intercambio de Científicos entre las universidades tradicionales y el servicio Alemán de intercambio académico (DAAD) que data de noviembre de 2003. Este programa de cooperación científica tiene como objetivo incrementar las relaciones de investigación en los campos científico - tecnológico, ciencias sociales, artes y humanidades entre Chile y Alemania.

2.- Bélgica: Con este país existe un convenio de intercambio académico entre Chile y Bélgica desde 1997, que ha sido complementado en el 2007 con la creación del Colegio Doctoral entre las universidades chilenas pertenecientes al Consejo de Rectores $\mathrm{CRUCH}^{7}$ - y las universidades del Consejo de Rectores de la Comunidad francesa de Bélgica - $\mathrm{CREF}^{8}$-. Este programa está dirigido a alumnos regulares de un doctorado en una universidad chilena perteneciente al CRUCH, que se encuentre en proceso de su tesis doctoral. Al finalizar su estadía, el alumno podrá acceder a la obtención de un doble grado académico, tanto de la universidad chilena de origen como de la Academia anfitriona en Bélgica.

3.- Brasil: El 9 de agosto de 2005 se firmó un Convenio de intercambio recíproco entre el Consejo de Rectores de las Universidades Brasileñas y su similar chileno con el objeto de “[...] cooperar en el ámbito de los estudios, de la docencia, de la investigación, mediante el intercambio de estudiantes, la admisión de estudiantes de doctorado, el intercambio de profesores e investigadores, y en la ejecución conjunta de proyectos de investigación" (Cláusula $1^{\mathrm{a}}$ del Convenio Marco).

4.- Canadá: El 22 de enero de 1998 se firmó un Memorándum de Entendimiento entre la Asociación de Universidades y Colegios de Canadá (AUCC) -que representa a 91 universidades canadienses- y el CRUCH -que representa a 25 universidades chilenas- con el objeto de promover la intemacionalización e intercambiar información en base a la trayectoria de cooperación que ha existido entre las instituciones de ambos países y dado los futuros prospectos de cooperación entre las comunidades científicas y académicas de Canadá y de Chile; para facilitar la relación entre ambas entidades habrá recursos para poder operar.

5.- España: En el año 2005 se estableció un Convenio entre el CRUCH y la Fundación Carolina con el objeto de financiar becas conjuntas de doctorado para alumnos chilenos en España. El compromiso de la Fundación Carolina es ofrecer a los postulantes oportunidades que garanticen una formación de calidad para que ellos puedan luego contribuir al desarrollo en su país de origen. Cada universidad, miembro del CRUCH podrá presentar la postulación de dos candidatos por convocatoria para las becas de 
doctorado y dos candidatos para estancias de investigación post - doctorales; dichas postulaciones deberán ir acompañadas de una carta compromiso de la respectiva universidad avalando la postulación del becario. En este momento está abierta la convocatoria 2010-2011.

6.- Francia: En el 2009 se renovó el Convenio Marco existente entre la Conferencia de Presidentes de las Universidades de Francia (CPU) y el CRUCH con la finalidad de " [...] la colaboración en los ámbitos de docencia, investigación y desarrollo, mediante el intercambio de estudiantes en los distintos niveles de estudios universitarios, el intercambio de docentes e investigadores, la realización de proyectos de investigación conjuntos y la consolidación en las relaciones de formación doctoral y post-doctoral" (Art. $1^{\circ}$ del Convenio Marco). El énfasis de este convenio es la formación de alto nivel académico para estudiantes chilenos para lo cual ambos países colaboran en el financiamiento. A su vez, Chile otorga becas para realizar pasantías de co-tutela en las universidades francesas que son parte del CPU por un período máximo de once meses contínuos. Este acuerdo de cooperación académica está especialmente dirigido a fomentar la co-tutela y la co-dirección de tesis doctorales. La co-tutela está orientada a la obtención del doble grado académico, una Universidad de Chile y su par en Francia. En el caso de la co-dirección, el estudiante obtiene el grado en una universidad.

7.- Italia: El 27 de febrero de 2002 se firmó el Acuerdo Marco de Cooperación Universitaria entre la Conferencia de Rectores de Italia (CRUI) y el CRUCH que tiene por objeto "[...] el intercambio de estudiantes de pre-grado y post-grado; el intercambio de académicos y asistentes de investigación, a fin de implementar proyectos en conjunto [...]; la elaboración de un sistema adecuado de transferencias de créditos universitarios [...]" (Art. 1 del Acuerdo Marco). Ambos países buscarán los mecanismos más adecuados para financiar estas actividades.

8.- Bolivia: Como una manera de producir un acercamiento entre Chile y Bolivia - que por razones de la pérdida del mar por parte de Bolivia en la guerra del Pacífico (1879), ambos países no tienen embajadores - durante los gobiernos de la Presidenta Michelle Bachelet y del Presidente Evo Morales se ha iniciado un diálogo que, entre otros aspectos, logró la firma de una Acta de Intenciones entre el CRUCH y el Comité Ejecutivo de la Universidad Boliviana el $1^{\circ}$ de septiembre de 2008; en él se pretende "[...] promover el intercambio de experiencias e iniciativas en el ámbito de la Enseñanza Superior y de la Investigación, que permitan contribuir a mejorar la calidad de vida de nuestros pueblos" (Considerando Acta de Intenciones).

El Ministerio de Educación de Chile tiene en su página web una información permanente sobre Educación Superior; allí, por ejemplo, aparece un link sobre "Movilidad Internacional de Estudiantes" y allí van apareciendo las diferentes ofertas de entidades extranjeras para estudiar en ellas con beca; en este momento aparecen "beca a Hong Kong" y "Beca a Taiwán". Los países de la cuenca del Pacífico y especialmente los más orientales desean un mayor intercambio con los países sudamericanos; Chile ha estado siendo visto como el país más adecuado para desde allí penetrar los mercados de los otros países. También aparece un link sobre "Movilidad Internacional de los académicos", en el cual se enfatizan los Convenios que implican Colegios Doctorales, como los existentes con Alemania y Francia, ya mencionados, y el Colegio Doctoral Argentina-Chile, cuya Carta de Intenciones entre la Secretaría de Políticas Universitarias del Ministerio de Educación 
de Argentina y la División de Educación Superior del MINEDUC se firmó en diciembre de 2008 , en que ambas partes se comprometieron a iniciar el trabajo de desarrollo e implementación de Colegios Doctorales Binacionales entre universidades de ambos países. Existe, finalmente un tercer link denominado "Movilidad de Programas e Instituciones"; allí aparece el Programa Pablo Neruda, en el cual participan siete países: Argentina, Chile, Colombia, Cuba, España, México y Portugal. Consiste en una acción de movilidad académica de postgrado de ambiente regional, sub-regional y multilateral. El objetivo general del programa Pablo Neruda es promover la construcción de un espacio iberoamericano del conocimiento (EIC) que favorezca la integración regional mediante la cooperación institucional. Las áreas temáticas definidas son Energía y Ciencias Ambientales, Biotecnología, Ciencias Agrarias, Ingenierías TICs y Bioingeniería, Desarrollo Social- Educación (www.mineduc/educacionsuperior.cl ).

El estado de Chile ha contraído hace algunos años, especialmente desde la vuelta a la democracia, un compromiso con los países de América Central, en el sentido de otorgar becas a alumnos de esos países que deseen proseguir estudios superiores en universidades chilenas.

Las universidades del Consejo de Rectores poseen, a su vez, sus propios Convenios. Considerando la gran cantidad de Convenios de Cooperación y/o de Intercambio académico, tanto para alumnos como para docentes, aquí se mencionarán sólo los países con los cuales en este momento existen Acuerdos de sólo dos universidades: las dos públicas más importantes y antiguas, la Universidad de Chile y la Universidad de Santiago de Chile. La Universidad de Chile tiene Convenios con universidades de Alemania, Argentina ${ }^{9}$, Australia, Austria, Bolivia, Brasil ${ }^{10}$, Canadá, Colombia, Corea, Costa Rica, Cuba, Dinamarca, Ecuador, España, Estados Unidos, Finlandia, Francia, Guatemala, Holanda, Hungría, Japón, México, Noruega, Nueva Zelandia, Panamá, Paraguay, Perú, Suecia, Suiza, Uruguay y Venezuela (www.udechile.cl ). La Universidad de Santiago de Chile, por su parte, ha firmado Convenios con universidades de Argentina $^{11}$, Austria, Bolivia, Brasi1 ${ }^{12}$, Canadá, China, Colombia, Costa Rica, Cuba, Ecuador, El Salvador, España, Estados Unidos, Francia, Honduras, Hungría, Inglaterra, Japón, Malasia, México, Nicaragua, Panamá, Paraguay, Perú, Polonia, Portugal, República Checa, Rumania, Rusia, Uruguay y Venezuela (www.usach.cl ).

Las universidades privadas también han firmado Convenios con universidades extranjeras. A modo de ejemplo, sólo nos referiremos a las ligadas con movimientos religiosos de carácter internacional. Ellas son la Universidad de Los Andes, ligada al Opus Dei, la Universidad Finis Terrea, ligada a los Legionarios de Cristo y la Universidad Alberto Hurtado, relacionada con la Compañía de Jesús.

La Universidad de Los Andes posee Convenios con universidades de Alemania, Argentina $^{13}$, Australia, Brasil ${ }^{14}$, Colombia, España ${ }^{15}$, Filipinas, Francia, Guatemala, Italia, México y Perú (www.ulosandes.cl ). La Universidad Finis Terrea ha firmado Convenios con universidades de Australia, Brasi1 ${ }^{16}$, Bulgaria, España, Estados Unidos y México ${ }^{17}$ (www.ufinisterrae.cl ). La Universidad Alberto Hurtado es parte de una red de más de 200 instituciones jesuitas de educación superior, presente en 65 países. Los miembros integrantes de esta red, son las Redes AUSJAL, de universidades latinoamericanas - está constituida por 31 universidades en 15 países de la región -, y la Red AJCU, de universidades y colleges estadounidenses - está constituida por 28 instituciones -. A abril de 2009 tenía 59 Convenios distribuidos en los siguientes países: Argentina ${ }^{18}$, Bolivia ${ }^{19}$, Brasil $^{20}$, Chile ${ }^{21}$, Colombia ${ }^{22}$, Ecuador ${ }^{23}$, El Salvador ${ }^{24}$, España ${ }^{25}$, Estados Unidos de América $^{26}$, Guatemala $^{27}$, México $^{28}$, Nicaragua ${ }^{29}$, Paraguay $^{30}$, Perú $^{31}$, República Dominicana $^{32}$, Suecia $^{33}$, Uruguay ${ }^{34}$ y Venezuela $^{35}$ (www.uah.cl ). 


\section{IV.- Esbozo de las principales estrategias usadas}

Al efectuar el rastreo de toda la información entregada precedentemente, es posible darse cuenta que los principales Convenios existen en su mayoría desde hace alrededor de 20 años, fecha que coincide con la vuelta a la democracia en el país; los Convenios de las universidades públicas chilenas se tiende a realizarlos con universidades públicas de otros países, justamente para afianzar la democracia y el desarrollo conjunto de los pueblos. Es decir, la estrategia más importante es buscar entidades análogas que persigan objetivos similares: Desarrollo de la ciencia y la tecnología e investigación científica, pero ligada al desarrollo de sus pueblos y a darles la movilidad social necesaria a los jóvenes. En efecto, en el pasado, sólo las personas de fortunas podían estudiar en otros países, especialmente europeos o norteamericanos; ahora, con la política de internacionalización de las universidades y el intercambio estudiantil entre ellas, las personas capaces de menores ingresos que han ingresado a la universidad pueden efectuar, especialmente estudios de post-grado en otros países. En Chile, por ejemplo, en los últimos años, 7 de cada 10 universitarios es la primera generación que lo logra, gracias a las políticas de mayor democratización de la educación.

Una segunda estrategia utilizada es unirse - en el caso de Chile en el Consejo de Rectores (CRUCH) - y buscar entidades análogas en otros países para firmar acuerdos Marco y, a partir de ellos, conocerse y firmar acuerdos específicos para determinadas áreas del saber.

Pero más importante que buscar las estrategias para internacionalizar la universidad, me parece significativo señalar las causas de aquello. El intercambio del saber ha sido a través de la historia algo consubstancial al quehacer universitario; sin embargo, la globalización y la apertura económica de las últimas décadas han favorecido el mayor intercambio académico; en el caso de Chile, uno de los países que posee un sistema económico con más apertura, tiene tratados de libre comercio con más de 56 países en todo el mundo y especialmente con la Unión Europea y con los Estados Unidos. Junto a los tratados de libre comercio han estado la firma de los Convenios de Cooperación e Intercambio Universitario.

La libertad académica y la autonomía universitaria han permitido, a su vez, el mayor desarrollo de la internacionalización de la educación superior, especialmente universitaria. Como consecuencia de aquello, en el caso de las universidades privadas, como se indicaron tres ejemplos en el punto anterior referidos a Chile, forman redes a través del mundo con entidades de igual o similar pensamiento, favoreciendo el intercambio académico, tanto de estudiantes como de académicos e investigadores.

\section{V.- Conclusión}

Para concluir, se puede señalar que en el mundo actual la internacionalización de la educación superior es una necesidad. La globalización, el libre comercio, la libre circulación de ideas y el afianzamiento de la democracia la han incentivado en los últimos decenios, haciéndola imprescindible para entidades que deseen tener altos estándares de calidad en la formación humana y el nivel académico de punta que el mundo de hoy requiere. 


\section{VI.- Anexos}

Anexo $\mathbf{N}^{\circ}$ 1: Universidades Tradicionales que conforman el Consejo de Rectores en Chile

\begin{tabular}{|c|c|c|c|c|}
\hline $\mathbf{N}$ & $\begin{array}{l}\text { UNIVERSIDADES } \\
\text { TRADICIONALES DEL } \\
\text { CONSEJO DE RECTORES }\end{array}$ & CIUDAD & $\begin{array}{l}\text { TIPO DE } \\
\text { INSTITUCION }\end{array}$ & $\begin{array}{l}\text { FECHA DE } \\
\text { RECONO- } \\
\text { CIMIENTO }\end{array}$ \\
\hline 1 & UNIVERSIDAD DE CHILE & SANTIAGO & ESTATAL & 19-11-1842 \\
\hline 2 & $\begin{array}{l}\text { UNIVERSIDAD DE SANTIAGO } \\
\text { DE CHILE }\end{array}$ & SANTIAGO & ESTATAL & 22-04-1947 \\
\hline 3 & $\begin{array}{l}\text { UNIVERSIDAD DE } \\
\text { VALPARAISO }\end{array}$ & VALPARAISO & ESTATAL & 12-02-1981 \\
\hline 4 & $\begin{array}{l}\text { UNIVERSIDAD DE } \\
\text { ANTOFAGASTA }\end{array}$ & ANTOFAGASTA & ESTATAL & 20-03-1981 \\
\hline 5 & UNIVERSIDAD DE LA SERENA & LA SERENA & ESTATAL & 20-03-1981 \\
\hline 6 & UNIVERSIDAD DEL BIO-BIO & CONCEPCION & ESTATAL & 20-03-1981 \\
\hline 7 & $\begin{array}{l}\text { UNIVERSIDAD DE LA } \\
\text { FRONTERA }\end{array}$ & TEMUCO & ESTATAL & 20-03-1981 \\
\hline 8 & $\begin{array}{l}\text { UNIVERSIDAD DE } \\
\text { MAGALLANES }\end{array}$ & PUNTA ARENAS & ESTATAL & 26-10-1981 \\
\hline 9 & UNIVERSIDAD DE TALCA & TALCA & ESTATAL & 26-10-1981 \\
\hline 10 & UNIVERSIDAD DE ATACAMA & COPIAPO & ESTATAL & 26-10-1981 \\
\hline 11 & UNIVERSIDAD DE TARAPACA & ARICA & ESTATAL & 18-01-1982 \\
\hline 12 & UNIVERSIDAD ARTURO PRAT & IQUIQUE & ESTATAL & $14-12-1984$ \\
\hline 13 & $\begin{array}{l}\text { UNIVERSIDAD } \\
\text { METROPOLITANA DE } \\
\text { CIENCIAS DE LA EDUCACION }\end{array}$ & SANTIAGO & ESTATAL & 04-09-1985 \\
\hline 14 & $\begin{array}{l}\text { UNIVERSIDAD DE PLAYA } \\
\text { ANCHA DE CIENCIAS DE LA } \\
\text { EDUCACION }\end{array}$ & VALPARAISO & ESTATAL & 04-09-1985 \\
\hline 15 & UNIVERSIDAD DE LOS LAGOS & OSORNO & ESTATAL & 30-08-1993 \\
\hline 16 & $\begin{array}{l}\text { UNIVERSIDAD TECNOLOGICA } \\
\text { METROPOLITANA }\end{array}$ & SANTIAGO & ESTATAL & 30-08-1993 \\
\hline 17 & $\begin{array}{l}\text { PONTIFICIA UNIVERSIDAD } \\
\text { CATOLICA DE CHILE }\end{array}$ & SANTIAGO & $\begin{array}{l}\text { U. } \\
\text { PARTICULARE } \\
\text { S CON } \\
\text { APORTE }\end{array}$ & $21-06-1888$ \\
\hline 18 & $\begin{array}{l}\text { UNIVERSIDAD DE } \\
\text { CONCEPCION }\end{array}$ & CONCEPCION & $\begin{array}{l}\text { U. } \\
\text { PARTICULARE } \\
\text { S CON } \\
\text { APORTE }\end{array}$ & $14-05-1920$ \\
\hline 19 & $\begin{array}{l}\text { UNIVERSIDAD TECNICA } \\
\text { FEDERICO SANTA MARIA }\end{array}$ & VALPARAISO & $\begin{array}{l}\text { U. } \\
\text { PARTICULARE } \\
\text { S CON } \\
\text { APORTE }\end{array}$ & $27-04-1926$ \\
\hline 20 & $\begin{array}{l}\text { PONTIFICIA UNIVERSIDAD } \\
\text { CATOLICA DE VALPARAISO }\end{array}$ & VALPARAISO & $\begin{array}{l}\text { U. } \\
\text { PARTICULARE } \\
\text { S CON } \\
\text { APORTE }\end{array}$ & $15-03-1928$ \\
\hline
\end{tabular}




\begin{tabular}{|l|l|l|l|l|}
21 & $\begin{array}{l}\text { UNIVERSIDAD AUSTRAL DE } \\
\text { CHILE }\end{array}$ & VALDIVIA & $\begin{array}{l}\text { U. } \\
\text { PARTICULARE } \\
\text { S CON } \\
\text { APORTE }\end{array}$ & $07-09-1954$ \\
\hline 22 & $\begin{array}{l}\text { UNIVERSIDAD CATOLICA DEL } \\
\text { NORTE }\end{array}$ & ANTOFAGASTA & $\begin{array}{l}\text { U. } \\
\text { PARTICULARE } \\
\text { S CON } \\
\text { APORTE }\end{array}$ & $01-01-1964$ \\
\hline 23 & $\begin{array}{l}\text { UNIVERSIDAD CATOLICA DEL } \\
\text { MAULE }\end{array}$ & TALCA & $\begin{array}{l}\text { U. } \\
\text { PARTICULARE } \\
\text { S CON } \\
\text { APORTE }\end{array}$ & $10-07-1991$ \\
\hline 24 & $\begin{array}{l}\text { UNIVERSIDAD CATOLICA DE } \\
\text { LA SANTISIMA CONCEPCION }\end{array}$ & CONCEPCION & $\begin{array}{l}\text { U. } \\
\text { PARTICULARE } \\
\text { S CON } \\
\text { APORTE }\end{array}$ & $10-07-1991$ \\
\hline 25 & $\begin{array}{l}\text { UNIVERSIDAD CATOLICA DE } \\
\text { TEMUCO }\end{array}$ & TEMUCO & $\begin{array}{l}\text { U. } \\
\text { PARTICULARE } \\
\text { S CON } \\
\text { APORTE }\end{array}$ & $10-07-1991$ \\
\hline
\end{tabular}

Fuente: División de Educación Superior, Mineduc

\section{Anexo No 2: Universidades Privadas en Chile}

\begin{tabular}{|c|c|c|c|}
\hline $\mathbf{N}^{\circ}$ UNIVERSIDADES PRIVADAS & CIUDAD & $\begin{array}{l}\text { TIPO DE } \\
\text { INSTITUCION }\end{array}$ & $\begin{array}{l}\text { FECHA DE } \\
\text { RECONOCIMIE } \\
\text { NTO }\end{array}$ \\
\hline 1)UNIVERSIDAD GABRIELA MISTRAL & SANTIAGO & U. PRIVADA & 01-12-1981 \\
\hline $\begin{array}{l}\text { 2UNIVERSIDAD FINIS TERRAE } \\
\text { UNIVR }\end{array}$ & SANTIAGO & U. PRIVADA & 02-01-1982 \\
\hline 3 UNIVERSIDAD DIEGO PORTALES & SANTIAGO & U. PRIVADA & $21-12-1982$ \\
\hline 4 UNIVERSIDAD CENTRAL DE CHILE & SANTIAGO & U. PRIVADA & $24-02-1983$ \\
\hline \begin{tabular}{l|l} 
UNIVERSIDAD BOLIVARIANA \\
\end{tabular} & SANTIAGO & U. PRIVADA & $21-06-1988$ \\
\hline 6UNIVERSIDAD PEDRO DE VALDIVIA & SANTIAGO & U. PRIVADA & $25-08-1988$ \\
\hline 7 UNIVERSIDAD MAYOR & SANTIAGO & U. PRIVADA & 13-05-1988 \\
\hline $8 \begin{array}{l}\text { UNIVERSIDAD ACADEMIA DE } \\
\text { HUMANISMO CRISTIANO }\end{array}$ & SANTIAGO & U. PRIVADA & $26-08-1988$ \\
\hline 9 UNIVERSIDAD SANTO TOMAS & SANTIAGO & U. PRIVADA & $26-12-1988$ \\
\hline 10|UNIVERSIDAD LA REPUBLICA & SANTIAGO & U. PRIVADA & 13-02-1989 \\
\hline 11|UNIVERSIDAD INTERNACIONAL SEK & SANTIAGO & U. PRIVADA & 09-02-1989 \\
\hline 12|UNIVERSIDAD DE LAS AMERICAS & SANTIAGO & U. PRIVADA & $23-12-1988$ \\
\hline $\begin{array}{ll}13 & \text { UNIVERSIDAD NACIONAL ANDRES } \\
\text { BELLO }\end{array}$ & SANTIAGO & U. PRIVADA & $29-12-1988$ \\
\hline 14|UNIVERSIDAD DE VIÑA DEL MAR & VIÑA DEL MAR & U. PRIVADA & $18-07-1989$ \\
\hline 15 UNIVERSIDAD ADOLFO IBAÑEZ & VIÑA DEL MAR & U. PRIVADA & 13-01-1989 \\
\hline $\begin{array}{l}\text { UNIVERSIDAD IBEROAMERICANA } \\
\text { 16E CIENCIAS Y TECNOLOGIA } \\
\text { UNICYT }\end{array}$ & SANTIAGO & U. PRIVADA & 25-09-1989 \\
\hline $\begin{array}{l}17 \text { UNIVERSIDAD DE ARTES, CIENCIAS } \\
\text { Y COMUNICACIÓN UNIACC }\end{array}$ & SANTIAGO & U. PRIVADA & 18-10-1989 \\
\hline 18UNIVERSIDAD DEL MAR & VIÑA DEL MAR & U. PRIVADA & 09-02-1990 \\
\hline $\begin{array}{l}19 \text { UNIVERSIDAD DE CIENCIAS DE LA } \\
\text { INFORMATICA }\end{array}$ & SANTIAGO & U. PRIVADA & $27-10-1989$ \\
\hline
\end{tabular}




\begin{tabular}{|c|c|c|c|}
\hline 20 UNIVERSIDAD AUTONOMA DE & TEMUCO & U. PRIVADA & 31-01-1990 \\
\hline 21 UNIVERSIDAD DE LOS ANDES & SANTIAGO & U. PRIVADA & 09-02-1990 \\
\hline $2 \begin{array}{l}\text { UNIVERSIDAD ADVENTISTA DE } \\
\text { CHILE }\end{array}$ & CHILLAN & U. PRIVADA & 06-02-1990 \\
\hline 23 UNIVERSIDAD SAN SEBASTIAN & CONCEPCION & U. PRIVADA & 09-02-1990 \\
\hline 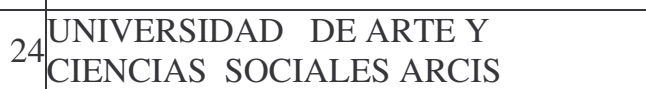 & SANTIAGO & U. PRIVADA & 09-03-1990 \\
\hline $\begin{array}{l}\text { UNIVERSIDAD CATOLICA } \\
25 \text { CARDENAL RAUL SILVA } \\
\text { HENRIQUEZ }\end{array}$ & SANTIAGO & U. PRIVADA & 16-08-1991 \\
\hline 26UNIVERSIDAD DEL DESARROLLO & CONCEPCION & U. PRIVADA & $22-02-1990$ \\
\hline 27 UNIVERSIDAD ACONCAGUA & SAN FELIPE & U. PRIVADA & 31-01-1991 \\
\hline 28|UNIVERSIDAD DEL PACIFICO & SANTIAGO & U. PRIVADA & 06-03-1990 \\
\hline 29UNIVERSIDAD MARITIMA DE CHILE & VIÑA DEL MAR & U. PRIVADA & 06-03-1990 \\
\hline $\begin{array}{l}30 \text { UNIVERSIDAD BERNARDO } \\
\text { O'HIGGINS }\end{array}$ & SANTIAGO & U. PRIVADA & 07-03-1990 \\
\hline \begin{tabular}{l|l}
31 & UNIVERSIDAD TECNOLOGICA DE \\
CHILE INACAP
\end{tabular} & SANTIAGO & U. PRIVADA & 29-01-1992 \\
\hline $\begin{array}{ll}32 & \text { UNIVERSIDAD MIGUEL DE } \\
\text { CERVANTES }\end{array}$ & SANTIAGO & U. PRIVADA & 27-11-1997 \\
\hline 33UNIVERSIDAD ALBERTO HURTADO & SANTIAGO & U. PRIVADA & 03-10-1997 \\
\hline 34UNIVERSIDAD DE RANCAGUA & RANCAGUA & U. PRIVADA & $11-12-2002$ \\
\hline $\begin{array}{l}35 \text { UNIVERSIDAD REGIONAL SAN } \\
\text { MARCOS }\end{array}$ & CONCEPCION & U. PRIVADA & 10-03-2004 \\
\hline $\begin{array}{l}36 \text { UNIVERSIDAD CHILENO BRITANICA } \\
\text { DE CULTURA }\end{array}$ & SANTIAGO & U. PRIVADA & $01-02-2006$ \\
\hline
\end{tabular}

Fuente: División de Educación Superior, Mineduc

\section{Notas:}

1 Participación en representación de Chile en el Panel "Estrategias de Internacionalización de la Educación Superior Universitaria" organizado por el Programa de Doctorado de la Universidad Nacional de Cuyo, Mendoza, Argentina, 27 de enero de 2010.

2 Académico de la Universidad de Santiago de Chile y del Doctorado de la Universidad Nacional de Cuyo, Mendoza, República Argentina..

3 Los títulos profesionales eran: Abogado, Arquitecto, Bioquímico, Cirujano Dentista, Ingeniero Agrónomo, Ingeniero Civil, Ingeniero Comercial, Ingeniero Forestal, Médico Cirujano, Médico Veterinario, Psicólogo y Químico Farmacéutico. Posteriormente, se incluyeron los títulos de Educadora de Párvulo, Pedagogía Básica y todas las Pedagogías de Enseñanza Media.

4 Esto se concretó sólo en 1991, con la vuelta a la democracia; durante la dictadura militar, la Iglesia Católica no permitió el desmembramiento de su principal Universidad Católica.

5 En Anexo № 1 se encuentra el listado de las mismas.

6 En Anexo № 2 se encuentra el listado de las mismas.

7 Sólo 13 de las 25 están habilitadas para otorgar el Grado Académico de Doctor: Universidad de Chile, Pontificia Universidad católica de Chile, Universidad de Santiago de Chile, Universidad de Concepción, Universidad Austral de Chile, Universidad Técnica Federico Santa María, Pontificia Universidad Católica de Valparaíso, Universidad de Valparaíso, Universidad Católica del Norte, Universidad de Tarapacá, Universidad de Talca, Universidad de Antofagasta y Universidad de La Frontera.

8 Entre ellas están: Université Catholique de Louvain, Université Libre de Bruxelles, Université de Liège, Université de Mons-Hainaut, Faculté Universitaire des Sciences Agronomiques de Gembloux, Facultés Universitaires Notre Dame de la Paix à Namur, Faculté Polytechnique de Mons, Facultés Universitaires Saint-Louis y Facultés Universitaires Catholiques de Mons. 
9 Los Convenios existentes, tanto marcos como específicos, son: Universidad de Buenos Aires, Universidad Católica de Cuyo, Universidad Nacional de Cuyo (marco y dos específicos: para la Facultad de Ciencias Químicas y Farmacéuticas y para la Facultad de Diseño y Arquitectura), Universidad de Mendoza, Universidad Nacional de Córdoba (marco y específico), Universidad Nacional de Salta, Universidad Nacional de San Juan (marco y específico), Universidad Nacional de Mar del Plata (marco y específico), Universidad Nacional de Jujuy, Universidad Nacional de La Plata, Universidad Nacional de Luján, Universidad Nacional de Rosario (marco y específico) y Universidad Nacional de Lanus.

10 Los Convenios existentes, tanto marcos como específicos, son: Universidad de Brasilia, Universidad de Sao Paulo (marco y específico), Universidad Federal de Paraná (específico), Universidad Federal de Río Grande do Sul, Universidad Federal Rural de Pernambuco, Universidad Federal de Minas Gerais, Universidad Estadual de Campinas, Universidad del Amazonas y Universidad Federal de Río de Janeiro.

11 Los Convenios existentes a la fecha son con las principales universidades públicas argentinas: Universidad de Buenos Aires, Universidad Nacional de Córdoba, Universidad Nacional de Misiones, Universidad Nacional de San Juan, Universidad Nacional del Nordeste, Universidad Nacional de Formosa, Universidad Nacional de La Plata, Universidad Nacional de Catamarca, Universidad Nacional de Lanus y Universidad Nacional de Cuyo; con ésta última existe un Convenio general -suscrito el 7 de enero de 1991 de colaboración cultural, científica y tecnológica- y uno específico -firmado el 18 de diciembre de 2008 con el objeto de efectuar intercambio de estudiantes entre ambas universidades-.

12 Los Convenios existentes, tanto generales como específicos para el intercambio de estudiantes son: Universidad Estadual de Maringá, Universidad Estadual de Campinas, Universidad Federal de Minas Gerais (marco y específico), Universidad Federal de Santa María, Universidad Santa Cecilia, Universidad de Sao Paulo (específico), Universidad Estadual Paulista "Julio de Mesquita Fihlo" (marco y específico) y Universidad de Santa Cruz do Sul,

13 Tiene Convenio con la Universidad de Salta y la Universidad Austral de Buenos Aires.

14 Posee Convenio con la Universidad Católica de Minas Gerais.

15 En este país nace el movimiento y, por lo mismo, hay Convenios con la Universidad de Navarra, Universidad de Santiago de Compostela, Universidad Católica de Valencia y Universidad de Salamanca.

16 En Brasil existe Convenio con la Universidad Federal de Bahía.

17 Aquí nació el movimiento y los Convenios existentes son: Universidad de La Salle en Ciudad de México y Universidad Tecnológica de Monterrey,

18 Facultades de Filosofía y Teología de San Miguel; Universidad Católica de Córdoba; Universidad de Cuyo; Universidad Nacional de Tucumán; La Universidad de Congreso; La Universidad Nacional del Nordeste y Universidad Católica de Santa Fe.

19 Instituto Superior de Filosofía y Humanidades Luis Espinal.

20 Centro Universitario da FEI; Facultade São Luis; Fundación Educacional Ignaciana Pe. Saboia de Mederios; Pontificia Universidad Católica de Rio de Janeiro; Universidad Católica de Pernambuco; Universidad do Vale do Rio de Sinos; Facultade Jesuita de Filosofía e Teología Faje-.

21 Pontificia Universidad Católica de Chile; Pontificia Universidad Católica de Valparaíso; Universidad Católica del Norte; Universidad Católica Silva Henríquez; Universidad Católica de la Santísima Concepción; Universidad Católica del Maule; Universidad Católica de Temuco y Universidad Diego Portales.

22 Pontificia Universidad Javeriana de Bogotá y Pontificia Universidad Javeriana de Calí.

23 Pontificia Universidad Católica de Ecuador.

24 Universidad Centroamericana José Simeón Cañas.

25 Universidad Autónoma de Madrid; Universidad de Deusto UNESCO y Pontificia Universidad de Comillas.

26 Boston College; Georgetown University; Loyola College in Maryland; Loyola University of Chicago; Santa Clara University; Saint Joseph's University; Saint Louis University; University of Notre Dame; University of San Francisco; University of Wisconsin Milwaukee y Mount Holyoke College, Massachusetts.

27 Universidad Rafael Landivar.

28 Instituto Tecnológico y de Estudios Superiores de Occidente; Universidad Iberoamericana Ciudad de México; Universidad Iberoamericana de Torreón; Universidad Iberoamericana León; 
Universidad Iberoamericana Puebla; Universidad Iberoamericana de Tijuana y Universidad Loyola del Pacífico.

29 Universidad Centroamericana.

30 Instituto Superior de Estudios Humanísticos y Filosóficos.

31 Universidad Antonio Ruiz de Montoya y Universidad del Pacífico.

32 Instituto Filosófico Pedro Francisco Bonó.

33 Newman Institute for Catholic Studies de Uppsala.

34 Universidad Católica de Uruguay.

35 Instituto Universitario Jesús Obrero; Universidad Católica Andrés Bello y Universidad Católica de Tachira.

Artigo recebido em: 20/03/2010

Aprovado em: 13/05/2010 